

\title{
Zigbee Based Smart Farm Data Logging and Monitoring System
}

\author{
Swe Swe Aung ${ }^{1}$, Naing Kyaw Soe ${ }^{2}$ \\ ${ }^{1}$ Assistant Lecturer, ${ }^{2}$ Lecturer \\ Department of Electronic Engineering, Pyay Technological University \\ Republic of the Union of Myanmar, Pyay (Western Bago), Myanmar
}

\section{ABSTRACT}

An agricultural environment monitoring system provides monitoring services and facility controlling services. This system maintains the crop growth rate in an optimal status. This system also reduces the manpower, time consumption and improves the convenience. The existing monitoring systems are used in indoor only or short distance wireless communication technology ( $\mathrm{Wi}-\mathrm{Fi}$ ) from sensor nodes to remote center. In addition, the users want to reduce the cost for checking the monitored information in Local Area Network of Smart Farm campus without using the internet access. In order to solve these issues, the agricultural monitoring system must be designed such a way that can monitor environmental information and soil information closely and reports the status to remote location in LAN. The main object of this research is to investigate the development of a low cost remote soil moisture monitoring system by deploying sensors, which can be used in a Zigbee mesh network. This sensors node send data via Zigbee transmitter wirelessly to a Zigbee receiver connected with main computer, which collects the data, stores it and will allow it to be analyzed. This data can be displayed as needed using GUI in VBA and can also be monitored by the clients in LAN.

Keyword: Arduino Microcontroller, Zigbee, Remote Monitoring, Smart Farm

\section{INTRODUCTION}

Agriculture is one of the most vital perspectives in human exercises around the world [1]. Agriculture is about the art of farming that contains growing of the crops through soil cultivating and providing foods, fleece and different products. Ever since the agriculture was born, farmers have been experienced such different problems in their everyday lives.
Farmers are facing new challenges on how they will retain their crops and animals to be healthy. Agriculture is highly dependent on the conditions of the climate. Soil moisture along with climatic conditions are the two most important factors, which decide the agriculture productivity. So, soil moisture levels in the field require periodic inspection, from where one can determine, when the next irrigation should be done and how much amount of water should be applied. Current developments in the miniaturization of electronic devices and wireless communication technology have resulted in the emergence of an energy efficient Wireless Sensor Networks (WSN). This makes it possible to acquire the field information more timely, accurately and conveniently. The sensors are designed for collecting information about the climatic condition of the smart farm like Temperature, Humidity and Soil Moisture. Monitoring parameters of temperature, humidity, and moisture is an important means for obtaining highquality environment. Remote monitoring is an effective method in order to avoid interference environment and improve efficiency. Today, Ethernet network and Zigbee wireless networks are used to transmit data in remote monitoring System. This paper gives not only a review of remote control and monitoring systems based on existing technologies but also a Zigbee based Local Area Network to allow efficient and low cost for smart farm data logging and monitoring.

\section{RELATED WORK}

Based on the review of related literature, the data logging and monitoring the data parameters within the field can be summarized as a common solution for the agricultural production. There are lots of different technology and methodology that can be used to 
monitor and $\log$ the data such as Wireless Sensor Network (WSN) technology, Smart Sensors Field Programmable Gate Array (FPGA)- based, Zigbee protocol microcontroller, Graphical User Interface, remote control through WSN, wireless monitoring with USB and transceiver chip, Zigbee protocol Arduino Microcontroller-based with global system for mobile communications. In synthesizing the overall related studies, the proponents did not such system which monitors, collect the data and logged all the information gathered through the Zigbee receiver with combined of GUI in VBA via Zigbee transmitter interfacing with Arduino microcontroller and sensors. The uniqueness of this study is that the system only covers the monitoring of the different parameters that have an effect to the quality and growth of the plants. In this system, the proponents focused to the monitoring and logging the data in Local Area Network of smart farm campus while in the related studies, the authors has a big coverage of their systems' functionality.

\section{PROPOSED SYSTEM}

In this system, the design and implementation of smart farm data logging and monitoring system evaluates the parameters needed in order to produce healthy crops.

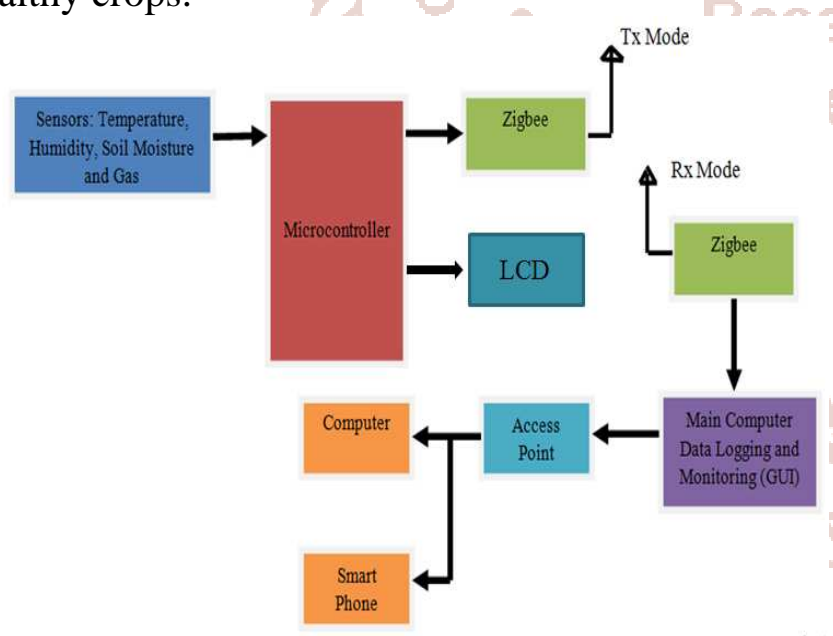

Figure1. Proposed System Design

This system monitors the temperature, humidity and soil moisture content as well. Signals are read by different sensors and its output is given to microcontroller. Output to microcontroller from sensor is taken and given to Zigbee. Signal is send to microcontroller and parameters like temperature, soil moisture and humidity are monitored. This data can be used for precision farming the actuators can be controlled using microcontroller data. The main system includes visual basic where the proponents will make the graphical user interface of the system which is easy to use and understand, and the Excel workbook that will serve as a database of the system. Furthermore, the data values of temperature, humidity and soil moisture will have a graphical representation to determine the possible abnormalities in a period of time. The whole system is made with the collaboration of hardware and software components. The hardware component includes Arduino UNO board, Sensors, Zigbee and Access Point. The software includes Arduino IDE, Smart RF Studio 7 and Termite.

\section{A. Humidity and Temperature Sensor DHT11}

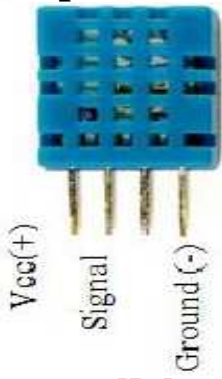

Figure2. DHT11 Temperature and Humidity Sensor

Humidity is the amount of water vapor present in the air. Humidity sensors detect the relative humidity of the surrounding environment. DHT11 measures both the humidity and temperature of the air. It expresses relative humidity as a percentage of the ratio of moisture in the air to the maximum amount that can be held in the air at the current temperature. Humidity sensors use capacitive method to find out the amount of moisture present in the air [2]. The temperature measurement is done using the principle of thermistor. This sensor provides a long term stability and high reliability. Also, it provides the advantages of high cost performances, quick result and a satisfaction of quality. This sensor can also monitor the humidity because it highlights an accurate calibration of humidity calibration chamber.

\section{B. Soil Moisture Sensor}

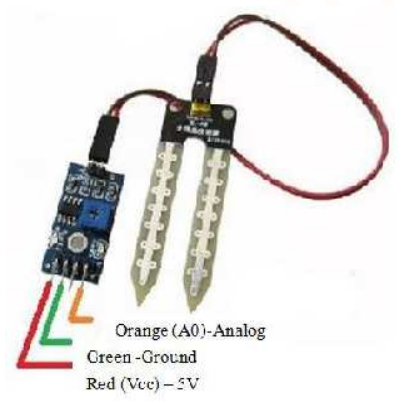

Figure3. Soil Moisture Sensor 
This sensor is used to evaluate the content of soil water level in volume unit. Due to the plant uptake and evaporation which leads to the loss of moisture, this sensor is capable in measuring its value. Also it can analyze the desired soil moisture contents for various species of crops. It can enhance the soil moisture content by monitoring and controlling the irrigation in farm [2]. The soil moisture sensor will be used by the proponents. This is the specific sensor that will detect the moisture level within the soil. It gives an accurate information of soil moisture content along with being cost-effective. This sensor can observe the moisture of the soil up to 10 meters above the ground.

\section{Arduino Microcontroller}

The Arduino UNO is a microcontroller board based on the ATmega 328. It has $6 \mathrm{MHz}$ ceramic resonator, a USB connector, reset button and a power jack. It is the most robust board. It has 14 digital I/O pins and ICSP header. Arduino does not require any extra hardware for boot loading. It is pre planned with boot loader that makes it simpler to upload programs to on board flash memory. The advantages of Arduino are that it provides inexpensive and simpler method for novices and professionals to create their own devices using sensors. It provides platform that easily runs on windows and Linux operating system.

\section{LCD (2x16)}

A liquid crystal display (LCD) is a thin, flat display device made up of any number of colour or monochrome pixels arrayed in front of a light source or reflector. Each pixel consists of a column of liquid crystal molecules suspended between two transparent electrodes, and two polarizing filters, the axes of polarity of which are perpendicular to each other. Many microcontroller devices use 'smart LCD' displays to output visual information.

\section{E. Zigbee}

Zigbee is developed by Zigbee alliance. It is based on IEEE 802.15.4 standards. Zigbee is well known wireless communication protocol. It has very less power consumption and is reliable for wireless personal area network. There are three types of device in Zigbee network. A network may consist of a coordinator node and multiple router and end devices. The configuration of Zigbee modules is done through Termite software [3]. Zigbee operates in the industrial, scientific and medical (ISM) radio bands: $915 \mathrm{MHz}$ in the USA and Australia, $868 \mathrm{MHz}$ in Europe, and $2.4 \mathrm{GHz}$ in worldwide. Apart from the agriculture and food industry, Zigbee is widely used in home building control, automation, security, consumer electronics, personal computer peripherals medical monitoring and toys [4]. Zigbee has following advantages in these applications such as, it offers long battery life, reliability, automatic or semiautomatic installation, the ability to easily add or remove network nodes, signals that can pass through walls and ceilings and a low system cost.

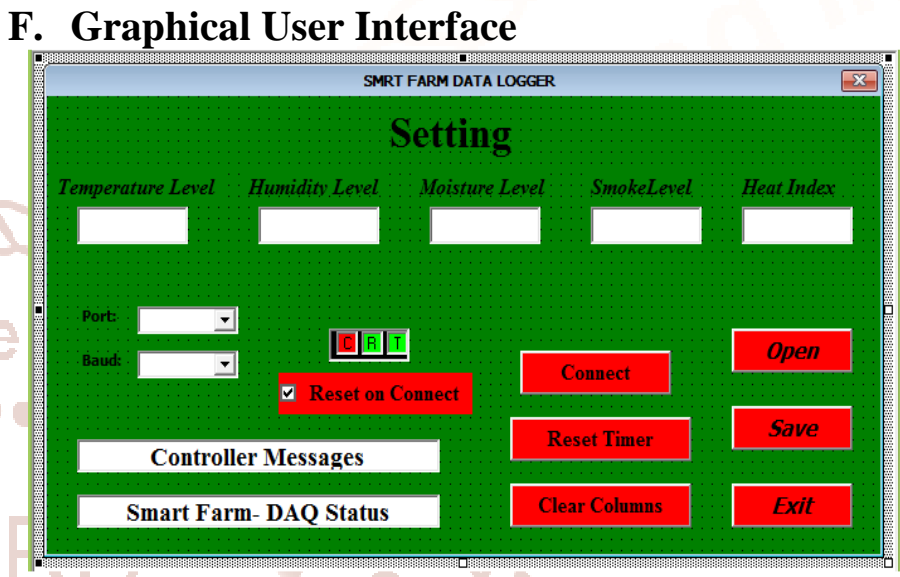

Figure4. Graphical User Interface of System

Figure 4 illustrated the graphical user interface design created in Visual Basic. This is used to monitor and $\log$ the data through the sensors.

\section{G. Visual Basic}

The Visual Basic will used by the proponents in implementing the system code. This programming language is much applicable to the design and implementation of the system because it has a best common language for improving the applications of windows [5].

\section{H. Access Point}

Access point connects users to other users within the network and also can serve as the point of interconnection between the WLAN and a fixed wire network. Each access point can serve multiple users within a defined network area.

\section{TEST AND RESULTS}

All sensors determined the soil moisture level, humidity and temperature at the root zone. This information is collected by the hardware controller and transmitted using Zigbee transmitter. On the other side all information are received by the Zigbee receiver which is store in the main system computer. Figure 5 and 6 are the hardware connection of Zigbee transmitter and sensors. 


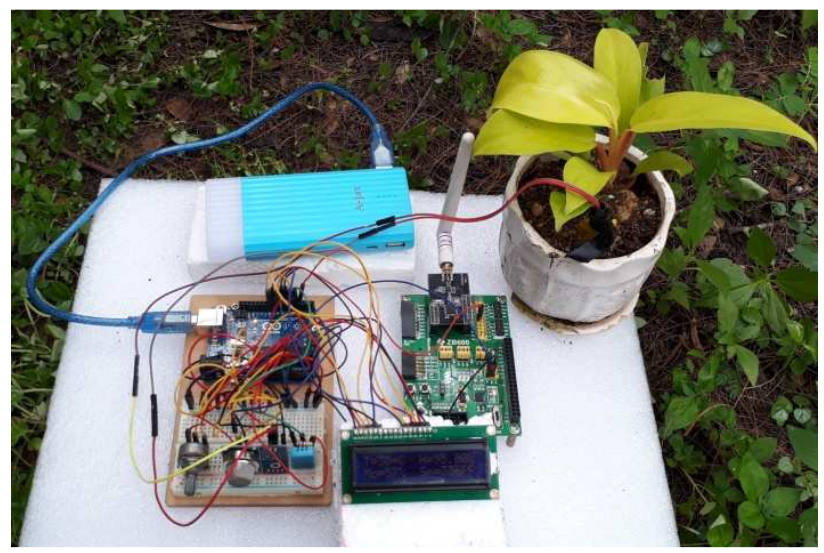

Figure5. Testing for Zigbee Transmitter and Sensors



Figure6. Sensor output display on LCD screen

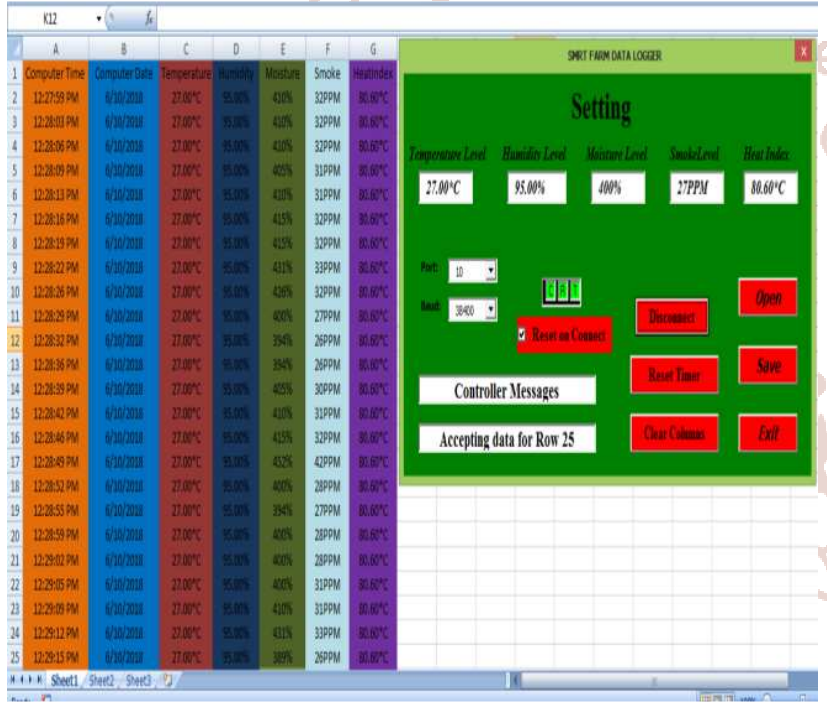

Figure7. Monitoring Sensor Data in Excel File

Figure 7 shows the sample of data values for temperature, humidity and soil moisture. This illustrates how the data values are being logged to excel database with respect to time and date. With this, the user can monitor the data gathered and will easily identify how data values changes. This data values gathered will be managed and analyzed by the other module of smart farm system if there are abnormalities encountered and will notify the farmers.
Humidity (\%)



Figure8. Graphical Representation of Humidity Level

Temperature( $\left.{ }^{\circ} \mathrm{C}\right)$

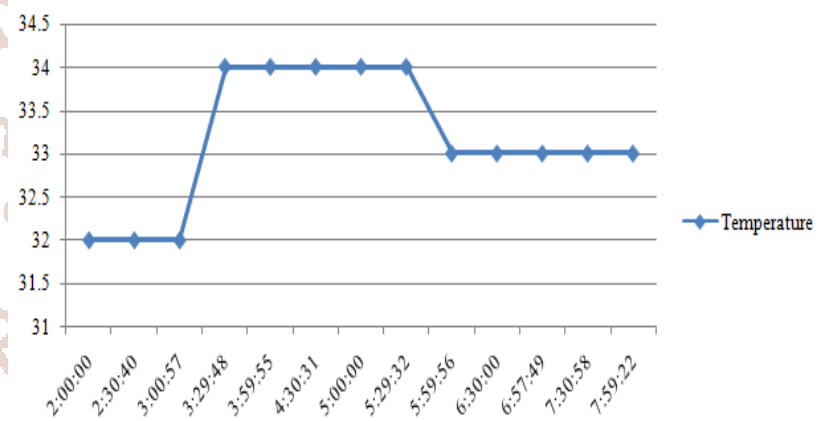

Figure9. Graphical Representation of Temperature Level

Moisture (\%)

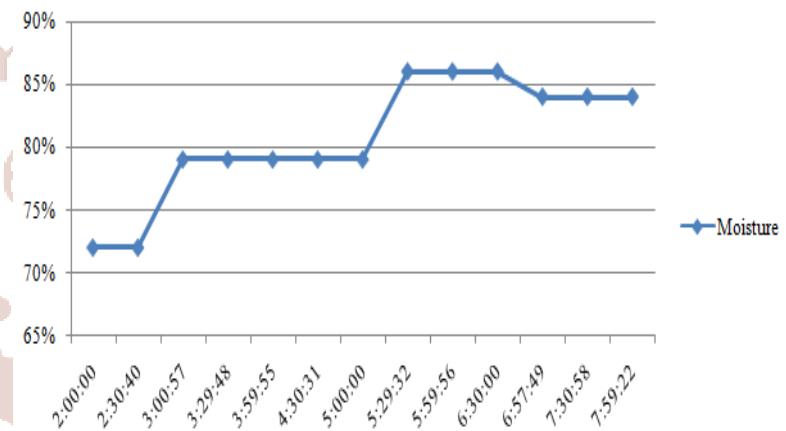

Figure10. Graphical Representation of Moisture Level

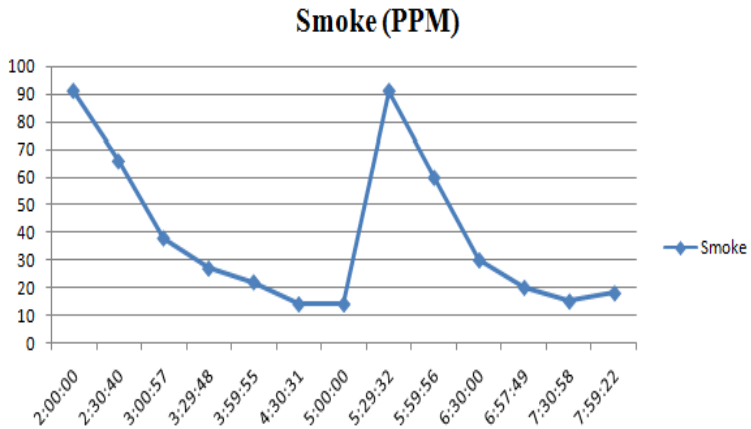

Figure11. Graphical Representation of Smoke Level 
Figure $8,9, \quad 10$, and 11 are the graphical representation for temperature, humidity, soil moisture. This paper will help to determine the abnormalities monitored by the system with that it can easily know the possible solution that needs to perform.

Figure 12 shows interfacing result data from Zigbee receiver mode

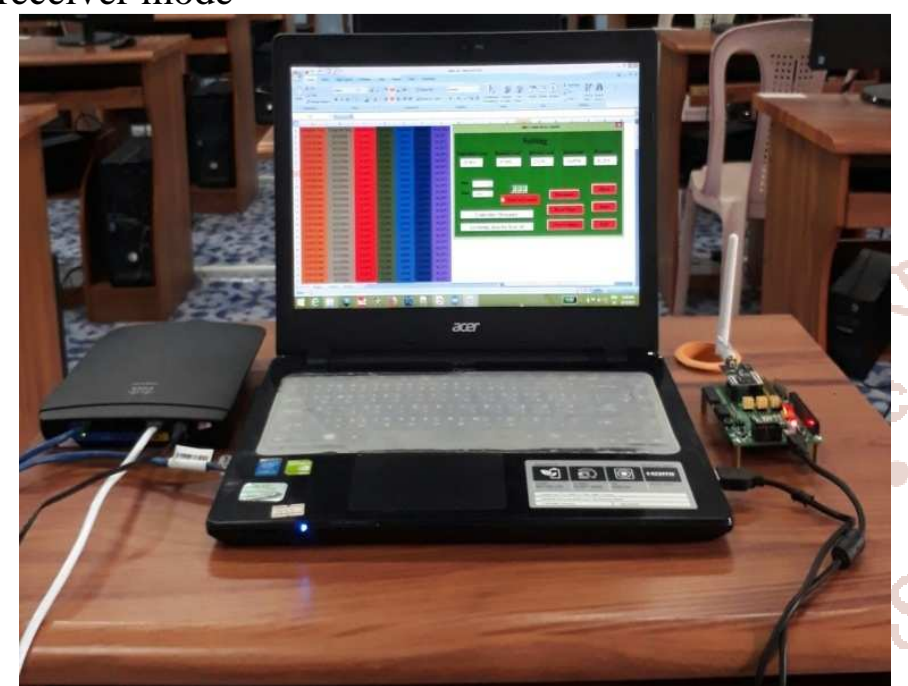

Figure12. Main Computer with Zigbee receiver

Figure 13 shows data is monitoring from mobile phone with smart farm campus.

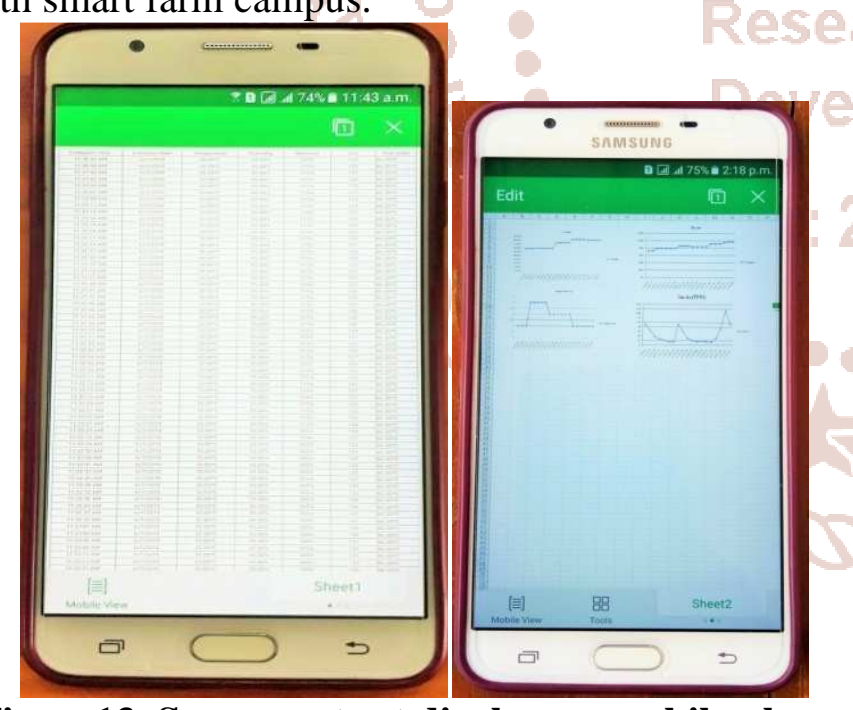

Figure13. Sensor output display on mobile phone

\section{CONCLUSION}

Zigbee-based agriculture monitoring system serves as a reliable and efficient system for monitoring agricultural parameters. Wireless monitoring of field will not only allow user to reduce the manual labor, but also to see accurate changes in it. It is cheaper in cost and consumes less power. Such a system can be easily installed and maintained. The scope for future work in this study will include fabrication, experimental investigation, and data analysis for predicting crop yield, control solution and complex networks setups. Moreover, this work can be extended to support different type of climatic condition to improve the growth rate and reduce the manpower using the automatic control system. The results can effective for the real application by deploying more sensor nodes with the additional sensors such as $\mathrm{CO} 2$ sensor and $\mathrm{pH}$ sensor.

\section{REFERENCES}

1. Online available at: https://www3.epa.gov/climatechange/impacts/agri culture.html/Accessed: 22- April- 2018.

2. (Ch. Apparao, G. Ravi Babu, A. Sambhaiah): Development of Low Cost Soil Moisture Sensor for Drip Irrigation System.

3. (Vellidis, G. Garrick, V. Pocknee, S. Perry, C. Kvien, C. and Tucker, M.): How wireless will change agriculture, Precision agriculture.

4. (G. V. Satyanarayana and S. D. Mazaruddin, 2015): Wireless Sensor Based Remote Monitoring System for Agriculture Using ZigBee and GPS.

5. Online available at: http://www.pcmag.com/encyclopedia/term/53685/ vb net/ Accessed: 6 - May-2018. 